CRYSTALLOGRAPHIC COMMUNICATIONS

ISSN 2056-9890

Received 6 July 2020

Accepted 19 July 2020

Edited by H. Ishida, Okayama University, Japan

Keywords: crystal structure; cycloaddition products; hexahydroimidazo[1,2-a]pyridine ring; SQUEEZE.

CCDC reference: 2017791

Supporting information: this article has supporting information at journals.iucr.org/e

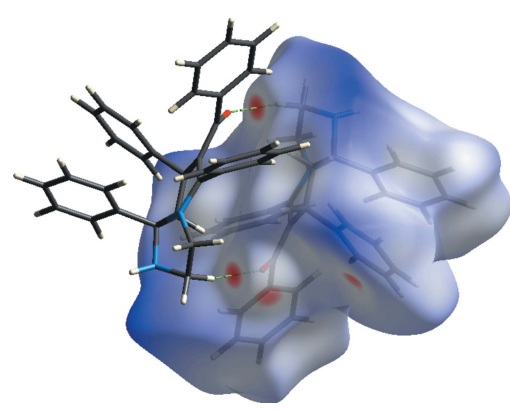

OPEN Ә ACCESS

\section{Crystal structure and Hirshfeld surface analysis of phenyl(5,7,8a-triphenyl-1,2,3,7,8,8a-hexahydro- imidazo[1,2-a]pyridin-6-yl)methanone with an unknown solvent}

Farid N. Naghiyev, ${ }^{a}$ Gunay Z. Mammadova, ${ }^{a}$ Ibrahim G. Mamedov, ${ }^{a}$ Afet T. Huseynova, ${ }^{a}$ Sevim Türktekin Celikesir, ${ }^{b}$ Mehmet Akkurt ${ }^{b}$ and Anzurat A. Akobirshoeva $^{\mathrm{C} *}$

${ }^{\mathbf{a}}$ Organic Chemistry Department, Baku State University, Z. Xalilov str. 23, Az, 1148 Baku, Azerbaijan, ${ }^{\mathbf{b}}$ Department of Physics, Faculty of Sciences, Erciyes University, 38039 Kayseri, Turkey, and 'Academy of Science of the Republic of Tadzhikistan, Kh. Yu. Yusufbekov Pamir Biological Institute, 1 Kholdorova St, Khorog 736002, Gbao, Tajikistan.

*Correspondence e-mail: anzurat2003@mail.ru

In the title compound, $\mathrm{C}_{32} \mathrm{H}_{28} \mathrm{~N}_{2} \mathrm{O}$, the imidazolidine and pyridine rings of the central hexahydroimidazo[1,2-a]pyridine ring system adopt envelope and screwboat conformations, respectively. The molecule exhibits two weak intramolecular $\pi-\pi$ interactions between phenyl rings. In the crystal, molecules are linked via pairs of $\mathrm{C}-\mathrm{H}$. . O hydrogen bonds, forming inversion dimers. The dimers are further linked by pairs of $\mathrm{C}-\mathrm{H} \cdots \pi$ interactions, forming infinite chains along the $c$-axis direction. A Hirshfeld surface analysis indicates that the most important contributions to the crystal packing are from $\mathrm{H} \cdots \mathrm{H}(73.4 \%)$, $\mathrm{C} \cdots \mathrm{H} / \mathrm{H} \cdots \mathrm{C}(18.8 \%)$ and $\mathrm{O} \cdots \mathrm{H} / \mathrm{H} \cdots \mathrm{O}(5.7 \%)$ contacts. The contribution of some disordered solvent to the scattering was removed using the SQUEEZE routine [Spek (2015). Acta Cryst. C71, 9-18] in PLATON. The solvent contribution was not included in the reported molecular weight and density.

\section{Chemical context}

Carbon-carbon and carbon-heteroatom bond-forming reactions are the most powerful and fundamental tools in synthetic organic chemistry. These synthetic approaches have successfully found applications in the construction of carbo- and heterocyclic ring systems (Khalilov et al., 2011; Yin et al., 2020). The use of nitrogen as the bridgehead atom is being assessed extensively. Bridgehead nitrogen heterocycles comprising imidazole rings are prevalent structural motifs in many compounds having applications in medicinal chemistry, coordination chemistry and material science (Afkhami et al., 2017; Mahmoudi et al., 2017a,b; Mahmudov et al., 2019, 2020). Various imidazo[1,2- $a$ ]pyridine moieties are included in synthetic drugs, such as alpidem, olprinone, saripidem, necopidem, miroprofen, zolimidine and zolpidem, which have already found use in medicinal practice. On the other hand, the imidazo[1,2-a]pyridine motif is also found in a series of natural products, such as oxaline and neoxaline (Koizumi et $a l ., 2004)$. As a result of the considerable interest to this field, there have been significant developments in the synthesis of imidazo[1,2-a]pyridine derivatives. In the framework of our ongoing structural studies (Akkurt et al., 2018; Khalilov et al., 2019), we report herein the crystal structure and Hirshfeld surface analysis of the title compound. 
<smiles>O=C(C1=C(c2ccccc2)N2CCNC2(c2ccccc2)CC1c1ccccc1)c1ccccc1</smiles>

\section{Structural commentary}

In the title compound (Fig. 1), the imidazolidine ring (N1/C1/ $\mathrm{C} 2 / \mathrm{N} 2 / \mathrm{C} 3)$ of the central hexahydroimidazo[1,2-a]pyridine ring system (N1/C1/C2/N2/C3-C7) adopts an envelope conformation with atom $\mathrm{C} 2$ as the flap lying 0.222 (2) $\AA$ from the mean plane of the remaining four atoms, while the pyridine ring $(\mathrm{N} 1 / \mathrm{C} 3-\mathrm{C} 7)$ is puckered with the puckering parameters $Q_{\mathrm{T}}=0.4970$ (15) $\AA, \theta=62.27(17)^{\circ}$ and $\varphi=96.49(19)^{\circ}$. The dihedral angles between phenyl rings are $A / B=34.51$ (8), $A / C=48.27(8), A / D=74.89(8), B / C=37.27(8), B / D=$

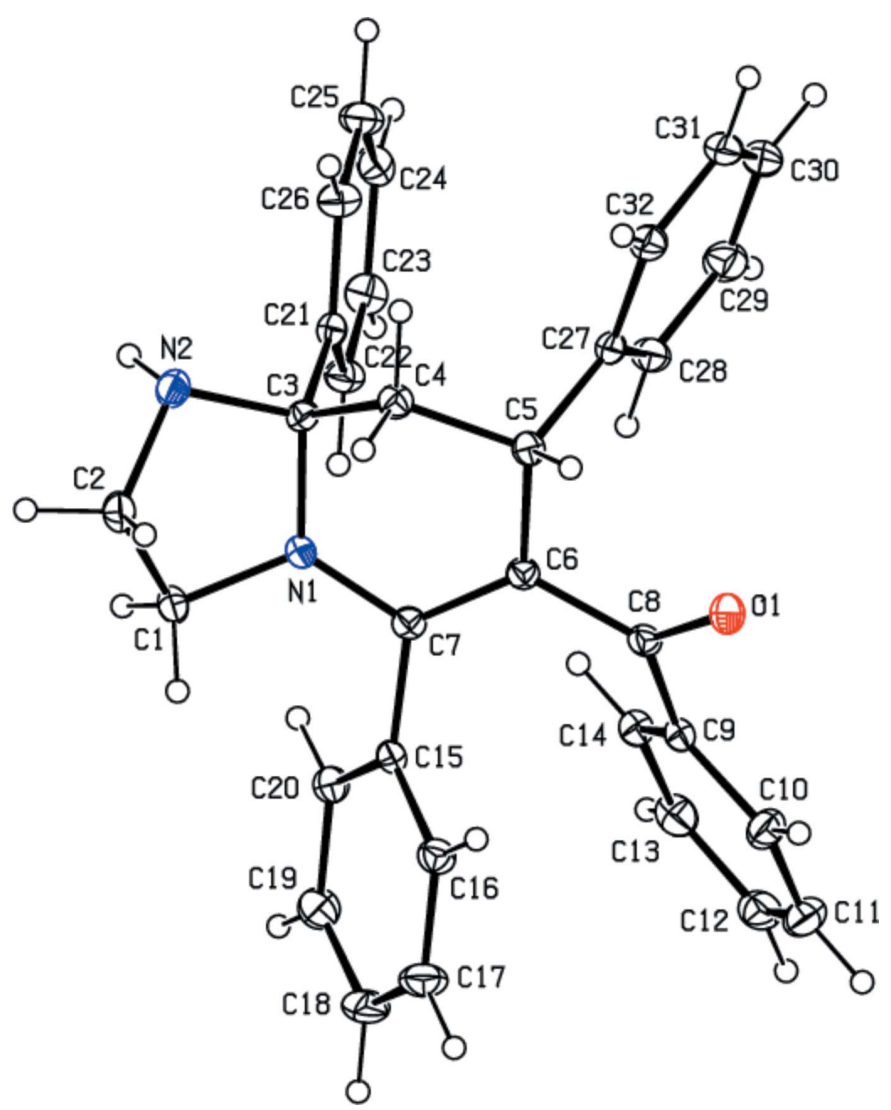

Figure 1

The molecular structure of the title compound, with displacement ellipsoids drawn at the $30 \%$ probability level.
Table 1

Hydrogen-bond geometry $\left(\AA,^{\circ}\right)$.

$\mathrm{Cg} 3$ is the centroid of the $\mathrm{C} 9-\mathrm{C} 14$ phenyl ring.

\begin{tabular}{lllll}
\hline$D-\mathrm{H} \cdots A$ & $D-\mathrm{H}$ & $\mathrm{H} \cdots A$ & $D \cdots A$ & $D-\mathrm{H} \cdots A$ \\
\hline $\mathrm{C} 2-\mathrm{H} 2 B \cdots \mathrm{O} 1^{\mathrm{i}}$ & 0.99 & 2.43 & $3.4084(19)$ & 168 \\
$\mathrm{C} 24-\mathrm{H} 24 \cdots C g 3^{\mathrm{ii}}$ & 0.95 & 2.83 & $3.5886(18)$ & 138 \\
\hline
\end{tabular}

Symmetry codes: (i) $-x,-y,-z+2$; (ii) $-x,-y,-z+1$.

56.29 (8) and $C / D=26.72(8)^{\circ}$, where $A, B, C$ and $D$ are the phenyl rings $\mathrm{C} 9-\mathrm{C} 14, \mathrm{C} 15-\mathrm{C} 20, \mathrm{C} 21-\mathrm{C} 26$ and $\mathrm{C} 27-\mathrm{C} 32$, respectively. The $A, B, C$ and $D$ ring planes are inclined to the central hexahydroimidazo[1,2-a]pyridine ring system, making dihedral angles of 60.24 (7), 61.73 (7), 81.91 (7) and 63.08 (7) respectively, with the mean plane of the central ring system. There are two weak intramolecular $\pi-\pi$ interactions $[C g 3 \cdots C g 4=3.7628(11) \AA$ and $C g 5 \cdots C g 6=3.9822(10) \AA$; $C g 3, C g 4, C g 5$ and $C g 6$ are the centroids of rings $A, B, C$ and $D$, respectively].

\section{Supramolecular features and Hirshfeld surface analysis}

In the crystal, molecules are linked via pairs of $\mathrm{C}-\mathrm{H} \cdots \mathrm{O}$ hydrogen bonds, forming inversion dimers. The dimers are further linked by pairs of $\mathrm{C}-\mathrm{H} \cdots \pi$ interactions, forming an infinite chain along the $c$-axis direction (Table 1 and Fig. 2).

In order to obtain further insight into the intermolecular interactions, we used Crystal Explorer (Turner et al., 2017). The Hirshfeld surface of the title compound mapped over $d_{\text {norm }}$ is depicted in Fig. 3, where the red regions are apparent around atom $\mathrm{O} 1$, which participates in the $\mathrm{C}-\mathrm{H} \cdots \mathrm{O}$ interactions (Table 1). The fingerprint plots (Fig. 4) show that the

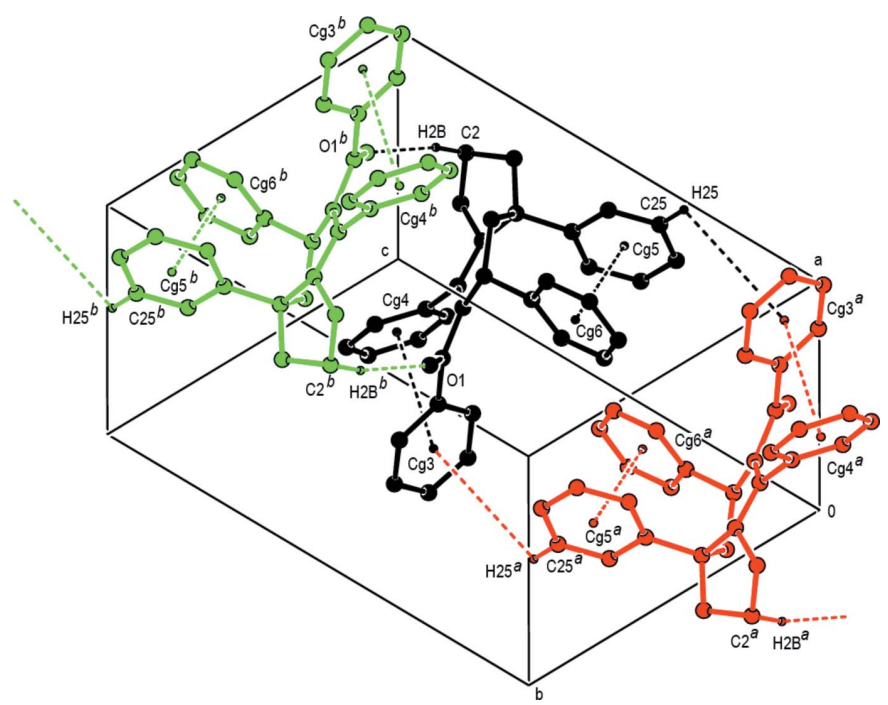

Figure 2

The crystal packing of the title compound. Dashed lines indicate $\mathrm{C}-$ $\mathrm{H} \cdots \mathrm{O}, \mathrm{C}-\mathrm{H} \cdots \pi$ and $\pi-\pi$ stacking interactions. $\mathrm{Cg} 3, \mathrm{Cg} 4, \mathrm{Cg} 5$ and $\mathrm{Cg} 6$ are the centroids of the C9-C14, C15-C20, C21-C26 and C27-C32 phenyl rings, respectively. [Symmetry codes: $(a)-x,-y,-z+1 ;(b)-x,-y$, $-z+2$. 


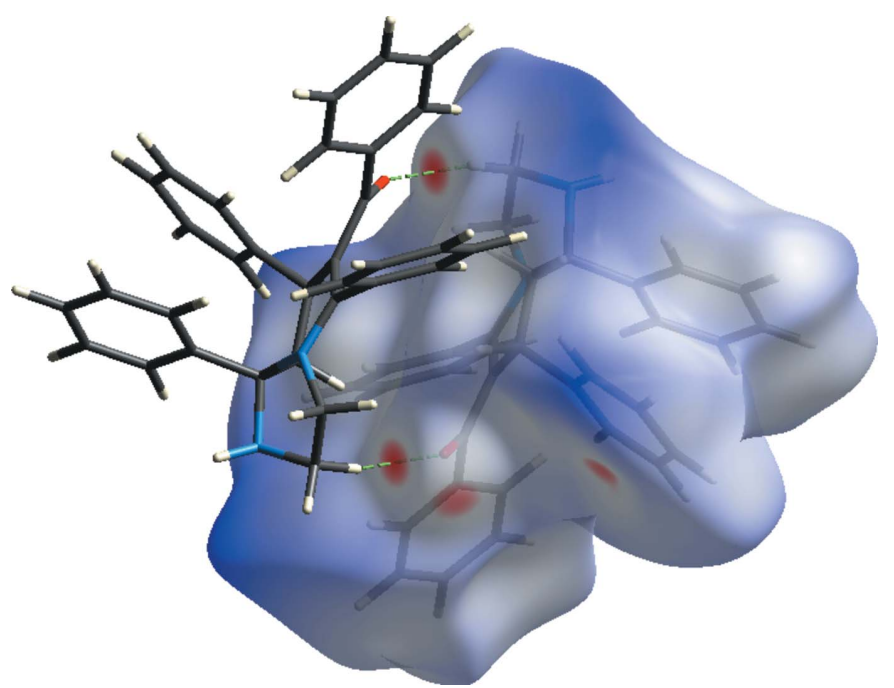

Figure 3

A view of the Hirshfeld surface of the title compound plotted over $d_{\text {norm }}$, showing the $\mathrm{C}-\mathrm{H} \cdots \mathrm{O}$ interactions.

largest contribution to the overall crystal packing is from $\mathrm{H} \cdot \mathrm{H}$ contacts $(73.4 \%)$. The second largest percentage $(18.8 \%)$ can be attributed to $\mathrm{C} \cdot \mathrm{H} / \mathrm{H} \cdots \mathrm{C}$ contacts, which correlate with the $\mathrm{C}-\mathrm{H} \cdots \pi$ interactions. $\mathrm{O} \cdots \mathrm{H} / \mathrm{H} \cdots \mathrm{O}$ contacts $(5.7 \%)$, which correlate with the $\mathrm{C}-\mathrm{H} \cdots \mathrm{O}$ interactions, provide another significant contribution to the Hirshfeld surface. Other contributions include $\mathrm{N} \cdots \mathrm{H} / \mathrm{H} \cdots \mathrm{N}$ $(1.9 \%)$ and $\mathrm{C} \cdots \mathrm{C}(0.2 \%)$. The removal of the contribution of the disordered solvent to the scattering using the SQUEEZE routine of $P L A T O N$ may be responsible for a small change in the given percentage contributions.

\section{Database survey}

A search in the Cambridge Structural Database (CSD, Version 5.41, updated to March 2020; Groom et al., 2016) gave three hits for the 1,2,3,7,8,8a-hexahydroimidazo[1,2-a]pyridine moiety, viz. 5,7,8a-triphenyl-1,2,3,7,8,8a-hexahydroimidazo[1,2-a]pyridine (KICJUE; Alvim et al., 2018), 7-(4-bromophenyl)-5,8a-diphenyl-1,2,3,7,8,8a-hexahydroimidazo[1,2-a]pyridine (TEZJOZ; Wang et al., 2013) and 8-benzyloxy-8amethyl-1,2,3,7,8,8a-hexahydroimidazo[1,2-a]pyridin-7-one monohydrate (YUYREP; Wireko et al., 1995). In KICJUE, single crystal $\mathrm{X}$-ray analysis confirmed the trans derivative as the only isomer. The structure of TEZJOZ shows that the aromatic ring of the aldehyde is on the other plane of the ketone in the purposed mechanism for the reaction. In the crystal of YUYREP, each water molecule bridges two molecules of the compound, hydrogen bonding with the carbonyl $\mathrm{O}$ atom of one molecule [O $\cdots \mathrm{O} W=2.796$ (4) $\AA$ ] and with the $\mathrm{N}$ atom of the other $[\mathrm{N} \cdots \mathrm{OW}=2.903$ (4) $\AA$ ]. The methyl group at the bridgehead is axially located in a trans position with respect to the bulky benzyloxy group. The pyridone ring assumes a slightly distorted half-chair conformation.

\section{Synthesis and crystallization}

To a solution of 2-benzoyl-1,3,5-triphenylpentane-1,5-dione $(3.5 \mathrm{mmol})$ in ethanol $(35 \mathrm{ml})$ was added ethylenediamine $(3.7 \mathrm{mmol})$ and 5 drops of concentrated $\mathrm{HCl}$. The mixture was stirred at room temperature for $15 \mathrm{~min}$, then refluxed for $4 \mathrm{~h}$ and cooled down to room temperature. The reaction product precipitated from the reaction mixture as colourless single crystals, which were collected by filtration and purified by recrystallization from ethanol (yield 76\%; m.p. 465-466 K).

${ }^{1} \mathrm{H}$ NMR $\left(300 \mathrm{MHz}, \mathrm{DMSO}-d_{6}\right): \delta 2.28\left(d d, 2 \mathrm{H}, \mathrm{CH}_{2} \mathrm{~N}\right)$, $2.77\left(d d, 2 \mathrm{H}, \mathrm{CH}_{2} \mathrm{~N}\right), 3.02(t, 1 \mathrm{H}, \mathrm{CH}), 3.41-3.63(d d, 2 \mathrm{H}$, $\left.\mathrm{CH}_{2}\right), 5.34(s, 1 \mathrm{H}, \mathrm{NH}), 6.82-7.78(m, 20 \mathrm{H}, 4 \mathrm{Ar}-\mathrm{H}) .{ }^{13} \mathrm{C} \mathrm{NMR}$ $\left(75 \mathrm{MHz}, \mathrm{DMSO}-d_{6}\right): \delta 37.63,45.55,48.71,48.98,75.80,125.99$, 126.72, 127.53, 128.06, 128.18, 128.43, 128.54, 128.99, 133.34, $136.99,145.47,146.49,170.71,199.38$.

\section{Refinement details}

Crystal data, data collection and structure refinement details are summarized in Table 2. The $\mathrm{N}$-bound $\mathrm{H}$ atom was located in a difference-Fourier map and refined freely $[\mathrm{N} 2-\mathrm{H} 2 \mathrm{~N}=$

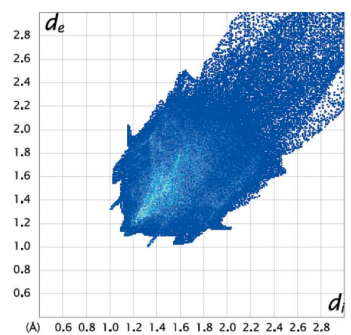

(a) All...All

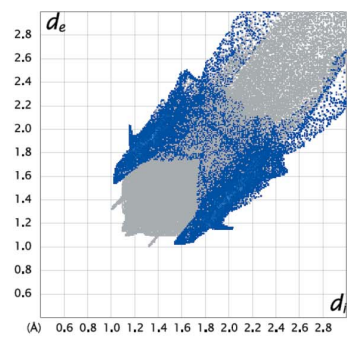

(c) $\mathrm{C} \ldots \mathrm{H} / \mathrm{H} . . . \mathrm{C}$

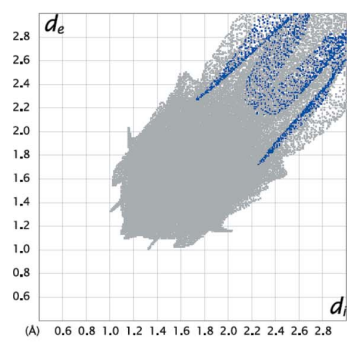

(e) $N \ldots H / H \ldots N$

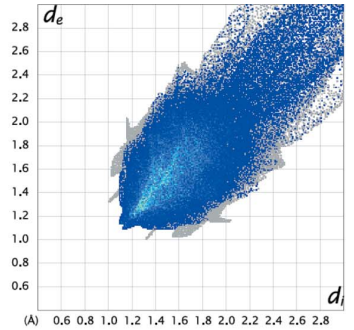

(b) $H \ldots H$

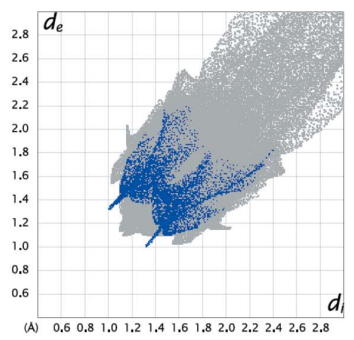

(d) $\mathrm{O} \ldots \mathrm{H} / \mathrm{H} \ldots \mathrm{O}$

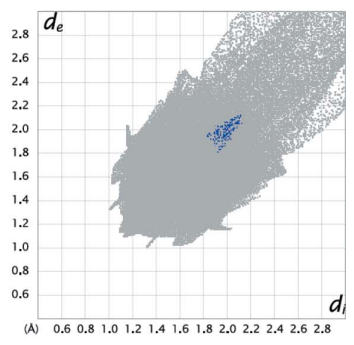

(f) $C \ldots C$

\section{Figure 4}

(a) A full two-dimensional fingerprint plot for the title compound, together with those delineated into $(b) \mathrm{H} \cdots \mathrm{H},(c) \mathrm{C} \cdots \mathrm{H} / \mathrm{H} \cdots \mathrm{C},(d)$ $\mathrm{O} \cdots \mathrm{H} / \mathrm{H} \cdots \mathrm{O},(e) \mathrm{N} \cdots \mathrm{H} / \mathrm{H} \cdots \mathrm{N}$ and $(f) \mathrm{C} \cdots \mathrm{C}$ contacts. 
0.908 (16) $\AA$ ]. The remaining $\mathrm{H}$ atoms were placed in calculated positions $(\mathrm{C}-\mathrm{H}=0.95-1.00 \AA)$ and allowed to ride on their carrier atoms, with $U_{\text {iso }}=1.2 U_{\text {eq }}(\mathrm{C})$. The residual electron density was difficult to model and therefore the SQUEEZE routine (Spek, 2015) in PLATON (Spek, 2020) was used to remove the contribution of the electron density in the solvent region from the intensity data and the solvent-free model was employed for the final refinement. The solvent formula mass and unit-cell characteristics were not taken into account during refinement. The cavity of volume $c a 119 \AA^{3}$ (ca $9.4 \%$ of the unit-cell volume) contains approximately 28 electrons.

\section{References}

Afkhami, F. A., Mahmoudi, G., Gurbanov, A. V., Zubkov, F. I., Qu, F., Gupta, A. \& Safin, D. A. (2017). Dalton Trans. 46, 14888-14896.

Akkurt, M., Duruskari, G. S., Toze, F. A. A., Khalilov, A. N. \& Huseynova, A. T. (2018). Acta Cryst. E74, 1168-1172.

Alvim, H. G. O., Correa, J. R., Assumpção, J. A. F., da Silva, W. A., Rodrigues, M. O., de Macedo, J. L., Fioramonte, M., Gozzo, F. C., Gatto, C. C. \& Neto, B. A. D. (2018). J. Org. Chem. 83, 4044-4053.

Bruker (2007). XSCANS . Bruker AXS Inc., Madison, Wisconsin, USA.

Farrugia, L. J. (2012). J. Appl. Cryst. 45, 849-854.

Groom, C. R., Bruno, I. J., Lightfoot, M. P. \& Ward, S. C. (2016). Acta Cryst. B72, 171-179.

Khalilov, A. N., Abdelhamid, A. A., Gurbanov, A. V. \& Ng, S. W. (2011). Acta Cryst. E67, o1146.

Khalilov, A. N., Atioğlu, Z., Akkurt, M., Duruskari, G. S., Toze, F. A. A. \& Huseynova, A. T. (2019). Acta Cryst. E75, 662-666.

Koizumi, Y., Arai, M., Tomoda, H. \& Ömura, S. (2004). Biochim. Biophys. Acta, 1693, 47-55.

Mahmoudi, G., Gurbanov, A. V., Rodríguez-Hermida, S., Carballo, R., Amini, M., Bacchi, A., Mitoraj, M. P., Sagan, F., Kukułka, M. \& Safin, D. A. (2017b). Inorg. Chem. 56, 9698-9709.

Mahmoudi, G., Zaręba, J. K., Gurbanov, A. V., Bauzá, A., Zubkov, F. I., Kubicki, M., Stilinović, V., Kinzhybalo, V. \& Frontera, A. (2017a). Eur. J. Inorg. Chem. 40, 4763-4772.

Mahmudov, K. T., Gurbanov, A. V., Aliyeva, V. A., Resnati, G. \& Pombeiro, A. J. L. (2020). Coord. Chem. Rev. 418, 213381.

Mahmudov, K. T., Gurbanov, A. V., Guseinov, F. I. \& Guedes da Silva, M. F. C. (2019). Coord. Chem. Rev. 387, 32-46.

Sheldrick, G. M. (1996). SADABS. University of Göttingen, Germany.

Sheldrick, G. M. (2008). Acta Cryst. A64, 112-122.

Sheldrick, G. M. (2015a). Acta Cryst. A71, 3-8.
Table 2

Experimental details.

\begin{tabular}{|c|c|}
\hline \multicolumn{2}{|l|}{ Crystal data } \\
\hline Chemical formula & $\mathrm{C}_{32} \mathrm{H}_{28} \mathrm{~N}_{2} \mathrm{O}$ \\
\hline$M_{\mathrm{r}}$ & 456.56 \\
\hline Crystal system, space group & Triclinic, $P \overline{1}$ \\
\hline Temperature (K) & 150 \\
\hline$a, b, c(\AA)$ & $\begin{array}{l}8.7807(9), 11.9566(12) \\
\quad 12.9121(13)\end{array}$ \\
\hline$\alpha, \beta, \gamma\left({ }^{\circ}\right)$ & 77.982 (1), 78.711 (1), $75.612(1)$ \\
\hline$V\left(\AA^{3}\right)$ & $1269.4(2)$ \\
\hline$Z$ & 2 \\
\hline Radiation type & Мo $K \alpha$ \\
\hline$\mu\left(\mathrm{mm}^{-1}\right)$ & 0.07 \\
\hline Crystal size $(\mathrm{mm})$ & $0.28 \times 0.25 \times 0.23$ \\
\hline \multicolumn{2}{|l|}{ Data collection } \\
\hline Diffractometer & Bruker P4 \\
\hline Absorption correction & $\begin{array}{l}\text { Multi-scan (SADABS; Sheldrick, } \\
\text { 1996) }\end{array}$ \\
\hline$T_{\min }, T_{\max }$ & $0.980,0.984$ \\
\hline $\begin{array}{l}\text { No. of measured, independent and } \\
\text { observed }[I>2 \sigma(I)] \text { reflections }\end{array}$ & $16061,6472,4328$ \\
\hline$R_{\text {int }}$ & 0.042 \\
\hline$(\sin \theta / \lambda)_{\max }\left(\AA^{-1}\right)$ & 0.695 \\
\hline \multicolumn{2}{|l|}{ Refinement } \\
\hline$R\left[F^{2}>2 \sigma\left(F^{2}\right)\right], w R\left(F^{2}\right), S$ & $0.051,0.117,1.03$ \\
\hline No. of reflections & 6472 \\
\hline No. of parameters & 320 \\
\hline $\mathrm{H}$-atom treatment & $\begin{array}{l}\mathrm{H} \text { atoms treated by a mixture of } \\
\text { independent and constrained } \\
\text { refinement }\end{array}$ \\
\hline$\Delta \rho_{\max }, \Delta \rho_{\min }\left(\mathrm{e} \AA^{-3}\right)$ & $0.25,-0.23$ \\
\hline
\end{tabular}

Computer programs: XSCANS (Bruker, 2007), SHELXTL (Sheldrick, 2008), SHELXT2014/5 (Sheldrick, 2015a), SHELXL2018/3 (Sheldrick, 2015b), ORTEP-3 for Windows (Farrugia, 2012) and PLATON (Spek, 2020).

Sheldrick, G. M. (2015b). Acta Cryst. C71, 3-8.

Spek, A. L. (2015). Acta Cryst. C71, 9-18.

Spek, A. L. (2020). Acta Cryst. E76, 1-11.

Turner, M. J., MacKinnon, J. J., Wolff, S. K., Grimwood, D. J., Spackman, P. R., Jayatilaka, D. \& Spackman, M. A. (2017). Crystal Explorer17.5. University of Western Australia. http://hirshfeldsurface.net.

Wang, R.-L., Zhu, P., Lu, Y., Huang, F.-P. \& Hui, X.-P. (2013). Adv. Synth. Catal. 355, 87-92.

Wireko, F. C., Matthews, R. S., Thoman, S. M., Hennes, D. H. \& Sickman, L. H. (1995). Acta Cryst. C51, 1404-1407.

Yin, J., Khalilov, A. N., Muthupandi, P., Ladd, R. \& Birman, V. B. (2020). J. Am. Chem. Soc. 142, 60-63. 


\section{supporting information}

Acta Cryst. (2020). E76, 1365-1368 [https://doi.org/10.1107/S2056989020009871]

Crystal structure and Hirshfeld surface analysis of phenyl(5,7,8a-triphenyl-1,2,3,7,8,8a-hexahydroimidazo[1,2-a]pyridin-6-yl)methanone with an unknown solvent

Farid N. Naghiyev, Gunay Z. Mammadova, Ibrahim G. Mamedov, Afet T. Huseynova, Sevim Türktekin Çelikesir, Mehmet Akkurt and Anzurat A. Akobirshoeva

Computing details

Data collection: XSCANS (Bruker, 2007); cell refinement: XSCANS (Bruker, 2007); data reduction: SHELXTL (Sheldrick, 2008); program(s) used to solve structure: SHELXT2014/5 (Sheldrick, 2015a); program(s) used to refine structure: SHELXL2018/3 (Sheldrick, 2015b); molecular graphics: ORTEP-3 for Windows (Farrugia, 2012); software used to prepare material for publication: PLATON (Spek, 2020).

Phenyl(5,7,8a-triphenyl-1,2,3,7,8,8a-hexahydroimidazo[1,2-a]pyridin-6-yl)methanone

Crystal data

$\mathrm{C}_{32} \mathrm{H}_{28} \mathrm{~N}_{2} \mathrm{O}$

$M_{r}=456.56$

Triclinic, $P \overline{1}$

$a=8.7807(9) \AA$

$b=11.9566(12) \AA$

$c=12.9121(13) \AA$

$\alpha=77.982(1)^{\circ}$

$\beta=78.711(1)^{\circ}$

$\gamma=75.612(1)^{\circ}$

$V=1269.4(2) \AA^{3}$

Data collection

Bruker P4

diffractometer

Radiation source: sealed tube

Graphite monochromator

$\omega$ scans

Absorption correction: multi-scan

(SADABS; Sheldrick, 1996)

$T_{\min }=0.980, T_{\max }=0.984$

Refinement

Refinement on $F^{2}$

Least-squares matrix: full

$R\left[F^{2}>2 \sigma\left(F^{2}\right)\right]=0.051$

$w R\left(F^{2}\right)=0.117$

$S=1.03$
$Z=2$

$F(000)=484$

$D_{\mathrm{x}}=1.195 \mathrm{Mg} \mathrm{m}^{-3}$

Mo $K \alpha$ radiation, $\lambda=0.71073 \AA$

Cell parameters from 2538 reflections

$\theta=2.2-24.2^{\circ}$

$\mu=0.07 \mathrm{~mm}^{-1}$

$T=150 \mathrm{~K}$

Prism, colourless

$0.28 \times 0.25 \times 0.23 \mathrm{~mm}$

16061 measured reflections

6472 independent reflections

4328 reflections with $I>2 \sigma(I)$

$R_{\text {int }}=0.042$

$\theta_{\text {max }}=29.6^{\circ}, \theta_{\text {min }}=1.8^{\circ}$

$h=-12 \rightarrow 12$

$k=-16 \rightarrow 16$

$l=-17 \rightarrow 17$

6472 reflections

320 parameters

0 restraints

Hydrogen site location: mixed 
$\mathrm{H}$ atoms treated by a mixture of independent and constrained refinement

$w=1 /\left[\sigma^{2}\left(F_{\mathrm{o}}^{2}\right)+(0.0474 P)^{2}+0.0165 P\right]$

where $P=\left(F_{\mathrm{o}}^{2}+2 F_{\mathrm{c}}^{2}\right) / 3$

$$
\begin{aligned}
& (\Delta / \sigma)_{\max }<0.001 \\
& \Delta \rho_{\max }=0.25 \mathrm{e} \AA^{-3} \\
& \Delta \rho_{\min }=-0.23 \mathrm{e} \AA^{-3}
\end{aligned}
$$

\section{Special details}

Geometry. All esds (except the esd in the dihedral angle between two 1.s. planes) are estimated using the full covariance matrix. The cell esds are taken into account individually in the estimation of esds in distances, angles and torsion angles; correlations between esds in cell parameters are only used when they are defined by crystal symmetry. An approximate

\begin{tabular}{|c|c|c|c|c|}
\hline & $x$ & $y$ & $z$ & $U_{\text {iso }} * / U_{\text {eq }}$ \\
\hline $\mathrm{C} 1$ & $0.44939(17)$ & $0.02866(13)$ & $0.83139(12)$ & $0.0254(3)$ \\
\hline $\mathrm{H} 1 \mathrm{~A}$ & 0.417532 & 0.092805 & 0.874131 & $0.031 *$ \\
\hline $\mathrm{H} 1 \mathrm{~B}$ & 0.532449 & 0.047058 & 0.770600 & $0.031 *$ \\
\hline $\mathrm{C} 2$ & 0.50577 (18) & -0.08820 & 0.89972 (12) & $0.0268(3)$ \\
\hline $\mathrm{H} 2 \mathrm{~A}$ & 0.620705 & -0.101848 & 0.903228 & $0.032 *$ \\
\hline $\mathrm{H} 2 \mathrm{~B}$ & 0.446137 & -0.091719 & 0.973388 & $0.032 *$ \\
\hline $\mathrm{C} 3$ & $0.32476(16)$ & $-0.11911(12)$ & $0.80213(11)$ & $0.0207(3)$ \\
\hline $\mathrm{C} 4$ & $0.18403(17)$ & $-0.15217(12)$ & $0.88356(11)$ & 0.0228 \\
\hline $\mathrm{H} 4 \mathrm{~A}$ & 0.188777 & -0.236789 & 0.889250 & $0.027^{*}$ \\
\hline $\mathrm{H} 4 \mathrm{~B}$ & 0.193319 & -0.138146 & 0.954753 & $0.027^{*}$ \\
\hline $\mathrm{C} 5$ & $0.02321(16)$ & $-0.08252(12)$ & $0.85304(11)$ & $0.0210(3)$ \\
\hline H5 & -0.054074 & -0.085719 & 0.921116 & $0.025^{*}$ \\
\hline C6 & $0.02583(16)$ & $0.04591(12)$ & $0.81466(11)$ & 0.0195 \\
\hline $\mathrm{C} 7$ & $0.16526(16)$ & $0.08404(12)$ & $0.80012(11)$ & 0.0198 \\
\hline $\mathrm{C} 8$ & $-0.13267(17)$ & $0.12259(12)$ & $0.81093(11)$ & 0.0215 \\
\hline C9 & $-0.16490(17)$ & $0.23969(12)$ & $0.74001(12)$ & 0.0229 \\
\hline $\mathrm{C} 10$ & $-0.29651(18)$ & $0.32328(14)$ & $0.77432(13)$ & $0.0316(4)$ \\
\hline $\mathrm{H} 10$ & -0.360310 & 0.305622 & 0.841463 & $0.038^{*}$ \\
\hline C11 & $-0.3348(2)$ & $0.43204(14)$ & $0.71104(14)$ & $0.0390(4)$ \\
\hline H11 & -0.423388 & 0.489185 & 0.735723 & $0.047 *$ \\
\hline $\mathrm{C} 12$ & $-0.2450(2)$ & $0.45780(14)$ & $0.61222(14)$ & $0.0373(4)$ \\
\hline H12 & -0.271383 & 0.532550 & 0.569140 & $0.045^{*}$ \\
\hline C13 & $-0.1165(2)$ & $0.37451(14)$ & $0.57624(13)$ & $0.0331(4)$ \\
\hline H13 & -0.055757 & 0.391481 & 0.507747 & $0.040 *$ \\
\hline $\mathrm{C} 14$ & $-0.07625(18)$ & $0.26622(13)$ & $0.63998(12)$ & $0.0257(3)$ \\
\hline H14 & 0.012783 & 0.209572 & 0.615064 & $0.031 *$ \\
\hline $\mathrm{C} 15$ & $0.17191(16)$ & $0.20907(12)$ & $0.78945(11)$ & 0.0206 \\
\hline $\mathrm{C} 16$ & $0.07779(18)$ & $0.27811(13)$ & $0.86290(12)$ & $0.0262(3)$ \\
\hline H16 & 0.010893 & 0.244616 & 0.922039 & $0.031^{*}$ \\
\hline $\mathrm{C} 17$ & $0.0815(2)$ & $0.39533(14)$ & $0.84988(14)$ & $0.0377(4)$ \\
\hline H17 & 0.017135 & 0.441920 & 0.900193 & $0.045^{*}$ \\
\hline $\mathrm{C} 18$ & $0.1783(2)$ & $0.44495(14)$ & $0.76422(15)$ & $0.0409(4)$ \\
\hline H18 & 0.179183 & 0.525745 & 0.754894 & $0.049 *$ \\
\hline C19 & $0.27404(19)$ & $0.37652(14)$ & $0.69207(14)$ & $0.0361(4)$ \\
\hline H19 & 0.341594 & 0.410217 & 0.633493 & $0.043 *$ \\
\hline
\end{tabular}
(isotropic) treatment of cell esds is used for estimating esds involving l.s. planes.

Fractional atomic coordinates and isotropic or equivalent isotropic displacement parameters $\left(\AA^{2}\right)$ 


$\begin{array}{lllll}\mathrm{C} 20 & 0.27169(17) & 0.25941(13) & 0.70494(12) & 0.0267(3) \\ \mathrm{H} 20 & 0.338866 & 0.212733 & 0.655604 & 0.032^{*} \\ \mathrm{C} 21 & 0.33725(16) & -0.15426(12) & 0.69346(11) & 0.0211^{(3)} \\ \mathrm{C} 22 & 0.34714(18) & -0.07518(13) & 0.59878(12) & 0.0264(3) \\ \mathrm{H} 22 & 0.341812 & 0.004668 & 0.600857 & 0.032^{*} \\ \mathrm{C} 23 & 0.36476(18) & -0.11116(14) & 0.50068(12) & 0.0306(4) \\ \mathrm{H} 23 & 0.371794 & -0.055874 & 0.436337 & 0.037^{*} \\ \mathrm{C} 24 & 0.37210(18) & -0.22659(14) & 0.49615(13) & 0.0297(4) \\ \mathrm{H} 24 & 0.382339 & -0.251013 & 0.429194 & 0.036^{*} \\ \mathrm{C} 25 & 0.36436(19) & -0.30625(13) & 0.59033(13) & 0.0322(4) \\ \mathrm{H} 25 & 0.370629 & -0.386175 & 0.587958 & 0.039^{*} \\ \mathrm{C} 26 & 0.34759(18) & -0.27082(13) & 0.68784(12) & 0.0292(4) \\ \mathrm{H} 26 & 0.343066 & -0.326753 & 0.751869 & 0.035^{*} \\ \mathrm{C} 27 & -0.03918(16) & -0.13787(12) & 0.77793(12) & 0.0221(3) \\ \mathrm{C} 28 & -0.05435(18) & -0.08759(13) & 0.67303(12) & 0.0275(3) \\ \mathrm{H} 28 & -0.024649 & -0.014775 & 0.644757 & 0.033^{*} \\ \mathrm{C} 29 & -0.1118(2) & -0.14096(15) & 0.60841(14) & 0.0363(4) \\ \mathrm{H} 29 & -0.120172 & -0.104929 & 0.536272 & 0.044^{*} \\ \mathrm{C} 30 & -0.15699(19) & -0.24589(15) & 0.64757(15) & 0.0378(4) \\ \mathrm{H} 30 & -0.197606 & -0.281885 & 0.603040 & 0.045^{*} \\ \mathrm{C} 31 & -0.14290(18) & -0.29836(14) & 0.75188(15) & 0.0350(4) \\ \mathrm{H} 31 & -0.172882 & -0.371193 & 0.779443 & 0.042^{*} \\ \mathrm{C} 32 & -0.08480(18) & -0.24458(13) & 0.81661(13) & 0.0292(4) \\ \mathrm{H} 32 & -0.075911 & -0.281070 & 0.888577 & 0.035^{*} \\ \mathrm{~N} 1 & 0.31103(13) & 0.00872(10) & 0.79395(9) & 0.0209(3) \\ \mathrm{N} 2 & 0.47413(15) & -0.17512(11) & 0.84545(11) & 0.0263(3) \\ \mathrm{H} 2 \mathrm{~N} & 0.553(2) & -0.1864(13) & 0.7890(13) & 0.033(4)^{*} \\ \mathrm{O} 1 & -0.24961(12) & 0.08722(9) & 0.86497(8) & 0.0286(3) \\ & & & & \end{array}$

Atomic displacement parameters $\left(\AA^{2}\right)$

\begin{tabular}{lllllll}
\hline & $U^{11}$ & $U^{22}$ & $U^{33}$ & $U^{12}$ & $U^{13}$ & $U^{23}$ \\
\hline C1 & $0.0190(8)$ & $0.0297(8)$ & $0.0306(8)$ & $-0.0071(6)$ & $-0.0052(6)$ & $-0.0079(7)$ \\
C2 & $0.0208(8)$ & $0.0331(9)$ & $0.0278(8)$ & $-0.0051(7)$ & $-0.0058(6)$ & $-0.0068(7)$ \\
C3 & $0.0187(7)$ & $0.0172(7)$ & $0.0251(8)$ & $-0.0023(6)$ & $-0.0034(6)$ & $-0.0029(6)$ \\
C4 & $0.0237(8)$ & $0.0212(7)$ & $0.0227(8)$ & $-0.0056(6)$ & $-0.0024(6)$ & $-0.0019(6)$ \\
C5 & $0.0191(7)$ & $0.0217(7)$ & $0.0214(7)$ & $-0.0069(6)$ & $0.0006(6)$ & $-0.0027(6)$ \\
C6 & $0.0199(7)$ & $0.0207(7)$ & $0.0186(7)$ & $-0.0049(6)$ & $-0.0011(6)$ & $-0.0055(6)$ \\
C7 & $0.0214(7)$ & $0.0222(7)$ & $0.0161(7)$ & $-0.0049(6)$ & $-0.0008(6)$ & $-0.0056(6)$ \\
C8 & $0.0209(7)$ & $0.0236(7)$ & $0.0222(7)$ & $-0.0060(6)$ & $-0.0010(6)$ & $-0.0095(6)$ \\
C9 & $0.0197(7)$ & $0.0240(8)$ & $0.0279(8)$ & $-0.0047(6)$ & $-0.0063(6)$ & $-0.0083(6)$ \\
C10 & $0.0263(9)$ & $0.0321(9)$ & $0.0346(9)$ & $0.0008(7)$ & $-0.0043(7)$ & $-0.0103(7)$ \\
C11 & $0.0374(10)$ & $0.0297(9)$ & $0.0488(11)$ & $0.0051(8)$ & $-0.0142(8)$ & $-0.0123(8)$ \\
C12 & $0.0440(11)$ & $0.0256(9)$ & $0.0463(11)$ & $-0.0066(8)$ & $-0.0222(9)$ & $-0.0016(7)$ \\
C13 & $0.0378(10)$ & $0.0328(9)$ & $0.0314(9)$ & $-0.0127(8)$ & $-0.0096(7)$ & $-0.0013(7)$ \\
C14 & $0.0241(8)$ & $0.0266(8)$ & $0.0282(8)$ & $-0.0055(6)$ & $-0.0058(6)$ & $-0.0068(6)$ \\
C15 & $0.0185(7)$ & $0.0211(7)$ & $0.0244(8)$ & $-0.0057(6)$ & $-0.0061(6)$ & $-0.0040(6)$ \\
C16 & $0.0271(8)$ & $0.0270(8)$ & $0.0264(8)$ & $-0.0075(7)$ & $-0.0023(6)$ & $-0.0080(6)$
\end{tabular}




$\begin{array}{lllllll}\text { C17 } & 0.0416(10) & 0.0283(9) & 0.0455(10) & -0.0085(8) & 0.0022(8) & -0.0182(8) \\ \text { C18 } & 0.0419(11) & 0.0203(8) & 0.0618(12) & -0.0120(7) & -0.0029(9) & -0.0083(8) \\ \text { C19 } & 0.0311(9) & 0.0282(9) & 0.0476(11) & -0.0130(7) & 0.0008(8) & -0.0013(8) \\ \text { C20 } & 0.0228(8) & 0.0256(8) & 0.0316(9) & -0.0072(6) & 0.0000(6) & -0.0058(7) \\ \text { C21 } & 0.0158(7) & 0.0230(7) & 0.0246(8) & -0.0039(6) & -0.0015(6) & -0.0057(6) \\ \text { C22 } & 0.0290(8) & 0.0226(8) & 0.0280(8) & -0.0082(6) & 0.0001(7) & -0.0059(6) \\ \text { C23 } & 0.0326(9) & 0.0338(9) & 0.0241(8) & -0.0094(7) & 0.0014(7) & -0.0047(7) \\ \text { C24 } & 0.0252(8) & 0.0390(9) & 0.0278(8) & -0.0104(7) & 0.0016(7) & -0.0134(7) \\ \text { C25 } & 0.0357(9) & 0.0250(8) & 0.0381(9) & -0.0093(7) & -0.0001(7) & -0.0120(7) \\ \text { C26 } & 0.0346(9) & 0.0232(8) & 0.0288(8) & -0.0061(7) & -0.0020(7) & -0.0050(6) \\ \text { C27 } & 0.0158(7) & 0.0209(7) & 0.0295(8) & -0.0034(6) & 0.0001(6) & -0.0078(6) \\ \text { C28 } & 0.0297(9) & 0.0244(8) & 0.0304(9) & -0.0072(7) & -0.0036(7) & -0.0083(6) \\ \text { C29 } & 0.0391(10) & 0.0367(10) & 0.0355(9) & -0.0026(8) & -0.0095(8) & -0.0148(8) \\ \text { C30 } & 0.0320(9) & 0.0373(10) & 0.0518(11) & -0.0033(8) & -0.0096(8) & -0.0263(9) \\ \text { C31 } & 0.0271(9) & 0.0233(8) & 0.0576(12) & -0.0075(7) & -0.0029(8) & -0.0143(8) \\ \text { C32 } & 0.0253(8) & 0.0228(8) & 0.0389(9) & -0.0061(6) & -0.0027(7) & -0.0045(7) \\ \text { N1 } & 0.0177(6) & 0.0212(6) & 0.0255(6) & -0.0057(5) & -0.0032(5) & -0.0061(5) \\ \text { N2 } & 0.0206(7) & 0.0289(7) & 0.0289(7) & -0.0016(5) & -0.0042(6) & -0.0080(6) \\ \text { O1 } & 0.0201(5) & 0.0320(6) & 0.0333(6) & -0.0078(5) & 0.0015(5) & -0.0069(5)\end{array}$

Geometric parameters $\left(\stackrel{A}{ }{ }^{o}\right)$

\begin{tabular}{llll}
\hline $\mathrm{C} 1-\mathrm{N} 1$ & $1.4794(18)$ & $\mathrm{C} 15-\mathrm{C} 20$ & $1.3910(19)$ \\
$\mathrm{C} 1-\mathrm{C} 2$ & $1.513(2)$ & $\mathrm{C} 15-\mathrm{C} 16$ & $1.3943(19)$ \\
$\mathrm{C} 1-\mathrm{H} 1 \mathrm{~A}$ & 0.9900 & $\mathrm{C} 16-\mathrm{C} 17$ & $1.384(2)$ \\
$\mathrm{C} 1-\mathrm{H} 1 \mathrm{~B}$ & 0.9900 & $\mathrm{C} 16-\mathrm{H} 16$ & 0.9500 \\
$\mathrm{C} 2-\mathrm{N} 2$ & $1.4736(18)$ & $\mathrm{C} 17-\mathrm{C} 18$ & $1.381(2)$ \\
$\mathrm{C} 2-\mathrm{H} 2 \mathrm{~A}$ & 0.9900 & $\mathrm{C} 17-\mathrm{H} 17$ & 0.9500 \\
$\mathrm{C} 2-\mathrm{H} 2 \mathrm{~B}$ & 0.9900 & $\mathrm{C} 18-\mathrm{C} 19$ & $1.383(2)$ \\
$\mathrm{C} 3-\mathrm{N} 2$ & $1.4752(18)$ & $\mathrm{C} 18-\mathrm{H} 18$ & 0.9500 \\
$\mathrm{C} 3-\mathrm{N} 1$ & $1.4859(17)$ & $\mathrm{C} 19-\mathrm{C} 20$ & $1.380(2)$ \\
$\mathrm{C} 3-\mathrm{C} 21$ & $1.5231(19)$ & $\mathrm{C} 19-\mathrm{H} 19$ & 0.9500 \\
$\mathrm{C} 3-\mathrm{C} 4$ & $1.5328(18)$ & $\mathrm{C} 20-\mathrm{H} 20$ & 0.9500 \\
$\mathrm{C} 4-\mathrm{C} 5$ & $1.530(2)$ & $\mathrm{C} 21-\mathrm{C} 22$ & $1.384(2)$ \\
$\mathrm{C} 4-\mathrm{H} 4 \mathrm{~A}$ & 0.9900 & $\mathrm{C} 21-\mathrm{C} 26$ & $1.3901(19)$ \\
$\mathrm{C} 4-\mathrm{H} 4 \mathrm{~B}$ & 0.9900 & $\mathrm{C} 22-\mathrm{C} 23$ & $1.389(2)$ \\
$\mathrm{C} 5-\mathrm{C} 6$ & $1.5166(18)$ & $\mathrm{C} 22-\mathrm{H} 22$ & 0.9500 \\
$\mathrm{C} 5-\mathrm{C} 27$ & $1.5279(19)$ & $\mathrm{C} 23-\mathrm{C} 24$ & $1.379(2)$ \\
$\mathrm{C} 5-\mathrm{H} 5$ & 1.0000 & $\mathrm{C} 23-\mathrm{H} 23$ & 0.9500 \\
$\mathrm{C} 6-\mathrm{C} 7$ & $1.3761(19)$ & $\mathrm{C} 24-\mathrm{C} 25$ & $1.381(2)$ \\
$\mathrm{C} 6-\mathrm{C} 8$ & $1.4675(19)$ & $\mathrm{C} 24-\mathrm{H} 24$ & 0.9500 \\
$\mathrm{C} 7-\mathrm{N} 1$ & $1.3685(17)$ & $\mathrm{C} 25-\mathrm{C} 26$ & $1.380(2)$ \\
$\mathrm{C} 7-\mathrm{C} 15$ & $1.4872(18)$ & $\mathrm{C} 25-\mathrm{H} 25$ & 0.9500 \\
$\mathrm{C} 8-\mathrm{O} 1$ & $1.2379(16)$ & $\mathrm{C} 26-\mathrm{H} 26$ & 0.9500 \\
$\mathrm{C} 8-\mathrm{C} 9$ & $1.500(2)$ & $\mathrm{C} 27-\mathrm{C} 28$ & $1.380(2)$ \\
$\mathrm{C} 9-\mathrm{C} 14$ & $1.390(2)$ & $\mathrm{C} 27-\mathrm{C} 32$ & $1.3957(19)$ \\
$\mathrm{C} 9-\mathrm{C} 10$ & $1.393(2)$ & $\mathrm{C} 28-\mathrm{C} 29$ & $1.380(2)$ \\
$\mathrm{C} 10-\mathrm{C} 11$ & $1.385(2)$ & $\mathrm{C} 28-\mathrm{H} 28$ & 0.9500 \\
- & &
\end{tabular}




\begin{tabular}{|c|c|c|c|}
\hline $\mathrm{C} 10-\mathrm{H} 10$ & 0.9500 & $\mathrm{C} 29-\mathrm{C} 30$ & $1.376(2)$ \\
\hline $\mathrm{C} 11-\mathrm{C} 12$ & $1.382(2)$ & $\mathrm{C} 29-\mathrm{H} 29$ & 0.9500 \\
\hline $\mathrm{C} 11-\mathrm{H} 11$ & 0.9500 & $\mathrm{C} 30-\mathrm{C} 31$ & $1.378(2)$ \\
\hline $\mathrm{C} 12-\mathrm{C} 13$ & $1.381(2)$ & $\mathrm{C} 30-\mathrm{H} 30$ & 0.9500 \\
\hline $\mathrm{C} 12-\mathrm{H} 12$ & 0.9500 & $\mathrm{C} 31-\mathrm{C} 32$ & $1.388(2)$ \\
\hline $\mathrm{C} 13-\mathrm{C} 14$ & $1.386(2)$ & $\mathrm{C} 31-\mathrm{H} 31$ & 0.9500 \\
\hline C13-H13 & 0.9500 & $\mathrm{C} 32-\mathrm{H} 32$ & 0.9500 \\
\hline C14-H14 & 0.9500 & $\mathrm{~N} 2-\mathrm{H} 2 \mathrm{~N}$ & $0.908(16)$ \\
\hline $\mathrm{N} 1-\mathrm{C} 1-\mathrm{C} 2$ & $101.91(11)$ & $\mathrm{C} 16-\mathrm{C} 15-\mathrm{C} 7$ & $120.90(12)$ \\
\hline $\mathrm{N} 1-\mathrm{C} 1-\mathrm{H} 1 \mathrm{~A}$ & 111.4 & $\mathrm{C} 17-\mathrm{C} 16-\mathrm{C} 15$ & $120.18(14)$ \\
\hline $\mathrm{C} 2-\mathrm{C} 1-\mathrm{H} 1 \mathrm{~A}$ & 111.4 & $\mathrm{C} 17-\mathrm{C} 16-\mathrm{H} 16$ & 119.9 \\
\hline $\mathrm{N} 1-\mathrm{C} 1-\mathrm{H} 1 \mathrm{~B}$ & 111.4 & $\mathrm{C} 15-\mathrm{C} 16-\mathrm{H} 16$ & 119.9 \\
\hline $\mathrm{C} 2-\mathrm{C} 1-\mathrm{H} 1 \mathrm{~B}$ & 111.4 & $\mathrm{C} 18-\mathrm{C} 17-\mathrm{C} 16$ & $120.41(15)$ \\
\hline $\mathrm{H} 1 \mathrm{~A}-\mathrm{C} 1-\mathrm{H} 1 \mathrm{~B}$ & 109.3 & $\mathrm{C} 18-\mathrm{C} 17-\mathrm{H} 17$ & 119.8 \\
\hline $\mathrm{N} 2-\mathrm{C} 2-\mathrm{C} 1$ & $104.60(12)$ & $\mathrm{C} 16-\mathrm{C} 17-\mathrm{H} 17$ & 119.8 \\
\hline $\mathrm{N} 2-\mathrm{C} 2-\mathrm{H} 2 \mathrm{~A}$ & 110.8 & $\mathrm{C} 17-\mathrm{C} 18-\mathrm{C} 19$ & $119.71(15)$ \\
\hline $\mathrm{C} 1-\mathrm{C} 2-\mathrm{H} 2 \mathrm{~A}$ & 110.8 & $\mathrm{C} 17-\mathrm{C} 18-\mathrm{H} 18$ & 120.1 \\
\hline $\mathrm{N} 2-\mathrm{C} 2-\mathrm{H} 2 \mathrm{~B}$ & 110.8 & $\mathrm{C} 19-\mathrm{C} 18-\mathrm{H} 18$ & 120.1 \\
\hline $\mathrm{C} 1-\mathrm{C} 2-\mathrm{H} 2 \mathrm{~B}$ & 110.8 & $\mathrm{C} 20-\mathrm{C} 19-\mathrm{C} 18$ & $120.19(15)$ \\
\hline $\mathrm{H} 2 \mathrm{~A}-\mathrm{C} 2-\mathrm{H} 2 \mathrm{~B}$ & 108.9 & $\mathrm{C} 20-\mathrm{C} 19-\mathrm{H} 19$ & 119.9 \\
\hline $\mathrm{N} 2-\mathrm{C} 3-\mathrm{N} 1$ & $105.07(11)$ & $\mathrm{C} 18-\mathrm{C} 19-\mathrm{H} 19$ & 119.9 \\
\hline $\mathrm{N} 2-\mathrm{C} 3-\mathrm{C} 21$ & $108.59(11)$ & $\mathrm{C} 19-\mathrm{C} 20-\mathrm{C} 15$ & $120.63(14)$ \\
\hline $\mathrm{N} 1-\mathrm{C} 3-\mathrm{C} 21$ & $112.32(11)$ & $\mathrm{C} 19-\mathrm{C} 20-\mathrm{H} 20$ & 119.7 \\
\hline $\mathrm{N} 2-\mathrm{C} 3-\mathrm{C} 4$ & $109.35(11)$ & $\mathrm{C} 15-\mathrm{C} 20-\mathrm{H} 20$ & 119.7 \\
\hline $\mathrm{N} 1-\mathrm{C} 3-\mathrm{C} 4$ & $107.36(11)$ & $\mathrm{C} 22-\mathrm{C} 21-\mathrm{C} 26$ & 118.31 \\
\hline $\mathrm{C} 21-\mathrm{C} 3-\mathrm{C} 4$ & $113.77(11)$ & $\mathrm{C} 22-\mathrm{C} 21-\mathrm{C} 3$ & $122.27(12)$ \\
\hline $\mathrm{C} 5-\mathrm{C} 4-\mathrm{C} 3$ & $112.67(11)$ & $\mathrm{C} 26-\mathrm{C} 21-\mathrm{C} 3$ & 119.33 \\
\hline $\mathrm{C} 5-\mathrm{C} 4-\mathrm{H} 4 \mathrm{~A}$ & 109.1 & $\mathrm{C} 21-\mathrm{C} 22-\mathrm{C} 23$ & $120.79(14)$ \\
\hline $\mathrm{C} 3-\mathrm{C} 4-\mathrm{H} 4 \mathrm{~A}$ & 109.1 & $\mathrm{C} 21-\mathrm{C} 22-\mathrm{H} 22$ & 119.6 \\
\hline $\mathrm{C} 5-\mathrm{C} 4-\mathrm{H} 4 \mathrm{~B}$ & 109.1 & $\mathrm{C} 23-\mathrm{C} 22-\mathrm{H} 22$ & 119.6 \\
\hline $\mathrm{C} 3-\mathrm{C} 4-\mathrm{H} 4 \mathrm{~B}$ & 109.1 & $\mathrm{C} 24-\mathrm{C} 23-\mathrm{C} 22$ & $120.40(15)$ \\
\hline $\mathrm{H} 4 \mathrm{~A}-\mathrm{C} 4-\mathrm{H} 4 \mathrm{~B}$ & 107.8 & $\mathrm{C} 24-\mathrm{C} 23-\mathrm{H} 23$ & 119.8 \\
\hline $\mathrm{C} 6-\mathrm{C} 5-\mathrm{C} 27$ & $114.32(12)$ & $\mathrm{C} 22-\mathrm{C} 23-\mathrm{H} 23$ & 119.8 \\
\hline $\mathrm{C} 6-\mathrm{C} 5-\mathrm{C} 4$ & $110.99(11)$ & $\mathrm{C} 23-\mathrm{C} 24-\mathrm{C} 25$ & $119.08(14)$ \\
\hline $\mathrm{C} 27-\mathrm{C} 5-\mathrm{C} 4$ & $113.18(11)$ & $\mathrm{C} 23-\mathrm{C} 24-\mathrm{H} 24$ & 120.5 \\
\hline $\mathrm{C} 6-\mathrm{C} 5-\mathrm{H} 5$ & 105.9 & $\mathrm{C} 25-\mathrm{C} 24-\mathrm{H} 24$ & 120.5 \\
\hline $\mathrm{C} 27-\mathrm{C} 5-\mathrm{H} 5$ & 105.9 & $\mathrm{C} 26-\mathrm{C} 25-\mathrm{C} 24$ & $120.62(14)$ \\
\hline $\mathrm{C} 4-\mathrm{C} 5-\mathrm{H} 5$ & 105.9 & $\mathrm{C} 26-\mathrm{C} 25-\mathrm{H} 25$ & 119.7 \\
\hline $\mathrm{C} 7-\mathrm{C} 6-\mathrm{C} 8$ & $124.93(12)$ & $\mathrm{C} 24-\mathrm{C} 25-\mathrm{H} 25$ & 119.7 \\
\hline $\mathrm{C} 7-\mathrm{C} 6-\mathrm{C} 5$ & $120.66(12)$ & $\mathrm{C} 25-\mathrm{C} 26-\mathrm{C} 21$ & $120.79(14)$ \\
\hline $\mathrm{C} 8-\mathrm{C} 6-\mathrm{C} 5$ & $113.82(12)$ & $\mathrm{C} 25-\mathrm{C} 26-\mathrm{H} 26$ & 119.6 \\
\hline $\mathrm{N} 1-\mathrm{C} 7-\mathrm{C} 6$ & $122.21(12)$ & $\mathrm{C} 21-\mathrm{C} 26-\mathrm{H} 26$ & 119.6 \\
\hline $\mathrm{N} 1-\mathrm{C} 7-\mathrm{C} 15$ & 114.14 (12) & $\mathrm{C} 28-\mathrm{C} 27-\mathrm{C} 32$ & $117.59(14)$ \\
\hline $\mathrm{C} 6-\mathrm{C} 7-\mathrm{C} 15$ & $123.64(12)$ & $\mathrm{C} 28-\mathrm{C} 27-\mathrm{C} 5$ & 123.52 \\
\hline $\mathrm{O} 1-\mathrm{C} 8-\mathrm{C} 6$ & $118.86(13)$ & $\mathrm{C} 32-\mathrm{C} 27-\mathrm{C} 5$ & $118.89(13)$ \\
\hline $\mathrm{O} 1-\mathrm{C} 8-\mathrm{C} 9$ & $116.85(13)$ & $\mathrm{C} 29-\mathrm{C} 28-\mathrm{C} 27$ & $121.33(15)$ \\
\hline $\mathrm{C} 6-\mathrm{C} 8-\mathrm{C} 9$ & $124.17(12)$ & $\mathrm{C} 29-\mathrm{C} 28-\mathrm{H} 28$ & 119.3 \\
\hline
\end{tabular}




\begin{tabular}{|c|c|c|c|}
\hline $\mathrm{C} 14-\mathrm{C} 9-\mathrm{C} 10$ & $118.77(14)$ & $\mathrm{C} 27-\mathrm{C} 28-\mathrm{H} 28$ & 119.3 \\
\hline $\mathrm{C} 14-\mathrm{C} 9-\mathrm{C} 8$ & $123.47(13)$ & $\mathrm{C} 30-\mathrm{C} 29-\mathrm{C} 28$ & $120.55(16)$ \\
\hline $\mathrm{C} 10-\mathrm{C} 9-\mathrm{C} 8$ & $117.65(13)$ & $\mathrm{C} 30-\mathrm{C} 29-\mathrm{H} 29$ & 119.7 \\
\hline $\mathrm{C} 11-\mathrm{C} 10-\mathrm{C} 9$ & $120.35(15)$ & $\mathrm{C} 28-\mathrm{C} 29-\mathrm{H} 29$ & 119.7 \\
\hline $\mathrm{C} 11-\mathrm{C} 10-\mathrm{H} 10$ & 119.8 & $\mathrm{C} 29-\mathrm{C} 30-\mathrm{C} 31$ & $119.46(15)$ \\
\hline $\mathrm{C} 9-\mathrm{C} 10-\mathrm{H} 10$ & 119.8 & $\mathrm{C} 29-\mathrm{C} 30-\mathrm{H} 30$ & 120.3 \\
\hline $\mathrm{C} 12-\mathrm{C} 11-\mathrm{C} 10$ & $120.33(16)$ & $\mathrm{C} 31-\mathrm{C} 30-\mathrm{H} 30$ & 120.3 \\
\hline $\mathrm{C} 12-\mathrm{C} 11-\mathrm{H} 11$ & 119.8 & $\mathrm{C} 30-\mathrm{C} 31-\mathrm{C} 32$ & $119.85(15)$ \\
\hline $\mathrm{C} 10-\mathrm{C} 11-\mathrm{H} 11$ & 119.8 & $\mathrm{C} 30-\mathrm{C} 31-\mathrm{H} 31$ & 120.1 \\
\hline $\mathrm{C} 13-\mathrm{C} 12-\mathrm{C} 11$ & $119.80(16)$ & $\mathrm{C} 32-\mathrm{C} 31-\mathrm{H} 31$ & 120.1 \\
\hline $\mathrm{C} 13-\mathrm{C} 12-\mathrm{H} 12$ & 120.1 & $\mathrm{C} 31-\mathrm{C} 32-\mathrm{C} 27$ & $121.21(16)$ \\
\hline $\mathrm{C} 11-\mathrm{C} 12-\mathrm{H} 12$ & 120.1 & $\mathrm{C} 31-\mathrm{C} 32-\mathrm{H} 32$ & 119.4 \\
\hline $\mathrm{C} 12-\mathrm{C} 13-\mathrm{C} 14$ & $120.08(16)$ & $\mathrm{C} 27-\mathrm{C} 32-\mathrm{H} 32$ & 119.4 \\
\hline $\mathrm{C} 12-\mathrm{C} 13-\mathrm{H} 13$ & 120.0 & $\mathrm{C} 7-\mathrm{N} 1-\mathrm{C} 1$ & $123.36(11)$ \\
\hline $\mathrm{C} 14-\mathrm{C} 13-\mathrm{H} 13$ & 120.0 & $\mathrm{C} 7-\mathrm{N} 1-\mathrm{C} 3$ & $120.74(11)$ \\
\hline $\mathrm{C} 13-\mathrm{C} 14-\mathrm{C} 9$ & $120.64(14)$ & $\mathrm{C} 1-\mathrm{N} 1-\mathrm{C} 3$ & $109.49(11)$ \\
\hline $\mathrm{C} 13-\mathrm{C} 14-\mathrm{H} 14$ & 119.7 & $\mathrm{C} 2-\mathrm{N} 2-\mathrm{C} 3$ & $105.65(11)$ \\
\hline C9- $14-\mathrm{H} 14$ & 119.7 & $\mathrm{C} 2-\mathrm{N} 2-\mathrm{H} 2 \mathrm{~N}$ & $107.9(10)$ \\
\hline $\mathrm{C} 20-\mathrm{C} 15-\mathrm{C} 16$ & $118.84(13)$ & $\mathrm{C} 3-\mathrm{N} 2-\mathrm{H} 2 \mathrm{~N}$ & $107.7(10)$ \\
\hline $\mathrm{C} 20-\mathrm{C} 15-\mathrm{C} 7$ & $120.26(12)$ & & \\
\hline $\mathrm{N} 1-\mathrm{C} 1-\mathrm{C} 2-\mathrm{N} 2$ & $34.54(14)$ & $\mathrm{N} 2-\mathrm{C} 3-\mathrm{C} 21-\mathrm{C} 22$ & $-110.98(15)$ \\
\hline $\mathrm{N} 2-\mathrm{C} 3-\mathrm{C} 4-\mathrm{C} 5$ & $170.85(11)$ & $\mathrm{N} 1-\mathrm{C} 3-\mathrm{C} 21-\mathrm{C} 22$ & $4.78(18)$ \\
\hline $\mathrm{N} 1-\mathrm{C} 3-\mathrm{C} 4-\mathrm{C} 5$ & $57.36(15)$ & $\mathrm{C} 4-\mathrm{C} 3-\mathrm{C} 21-\mathrm{C} 22$ & $127.01(14)$ \\
\hline $\mathrm{C} 21-\mathrm{C} 3-\mathrm{C} 4-\mathrm{C} 5$ & $-67.56(15)$ & $\mathrm{N} 2-\mathrm{C} 3-\mathrm{C} 21-\mathrm{C} 26$ & $65.54(16)$ \\
\hline $\mathrm{C} 3-\mathrm{C} 4-\mathrm{C} 5-\mathrm{C} 6$ & $-43.64(15)$ & $\mathrm{N} 1-\mathrm{C} 3-\mathrm{C} 21-\mathrm{C} 26$ & $-178.70(12)$ \\
\hline $\mathrm{C} 3-\mathrm{C} 4-\mathrm{C} 5-\mathrm{C} 27$ & $86.46(14)$ & $\mathrm{C} 4-\mathrm{C} 3-\mathrm{C} 21-\mathrm{C} 26$ & $-56.47(17)$ \\
\hline $\mathrm{C} 27-\mathrm{C} 5-\mathrm{C} 6-\mathrm{C} 7$ & $-122.71(14)$ & $\mathrm{C} 26-\mathrm{C} 21-\mathrm{C} 22-\mathrm{C} 23$ & $0.9(2)$ \\
\hline $\mathrm{C} 4-\mathrm{C} 5-\mathrm{C} 6-\mathrm{C} 7$ & $6.78(18)$ & $\mathrm{C} 3-\mathrm{C} 21-\mathrm{C} 22-\mathrm{C} 23$ & $177.43(14)$ \\
\hline $\mathrm{C} 27-\mathrm{C} 5-\mathrm{C} 6-\mathrm{C} 8$ & $65.67(15)$ & $\mathrm{C} 21-\mathrm{C} 22-\mathrm{C} 23-\mathrm{C} 24$ & $0.2(2)$ \\
\hline $\mathrm{C} 4-\mathrm{C} 5-\mathrm{C} 6-\mathrm{C} 8$ & $-164.83(11)$ & $\mathrm{C} 22-\mathrm{C} 23-\mathrm{C} 24-\mathrm{C} 25$ & $-1.1(2)$ \\
\hline $\mathrm{C} 8-\mathrm{C} 6-\mathrm{C} 7-\mathrm{N} 1$ & $-173.38(13)$ & $\mathrm{C} 23-\mathrm{C} 24-\mathrm{C} 25-\mathrm{C} 26$ & $0.8(2)$ \\
\hline $\mathrm{C} 5-\mathrm{C} 6-\mathrm{C} 7-\mathrm{N} 1$ & $16.0(2)$ & $\mathrm{C} 24-\mathrm{C} 25-\mathrm{C} 26-\mathrm{C} 21$ & $0.4(2)$ \\
\hline $\mathrm{C} 8-\mathrm{C} 6-\mathrm{C} 7-\mathrm{C} 15$ & $6.4(2)$ & $\mathrm{C} 22-\mathrm{C} 21-\mathrm{C} 26-\mathrm{C} 25$ & $-1.2(2)$ \\
\hline $\mathrm{C} 5-\mathrm{C} 6-\mathrm{C} 7-\mathrm{C} 15$ & $-164.19(12)$ & $\mathrm{C} 3-\mathrm{C} 21-\mathrm{C} 26-\mathrm{C} 25$ & $-177.83(14)$ \\
\hline $\mathrm{C} 7-\mathrm{C} 6-\mathrm{C} 8-\mathrm{O} 1$ & $-150.77(14)$ & $\mathrm{C} 6-\mathrm{C} 5-\mathrm{C} 27-\mathrm{C} 28$ & $15.65(19)$ \\
\hline $\mathrm{C} 5-\mathrm{C} 6-\mathrm{C} 8-\mathrm{O} 1$ & $20.43(18)$ & $\mathrm{C} 4-\mathrm{C} 5-\mathrm{C} 27-\mathrm{C} 28$ & $-112.74(15)$ \\
\hline $\mathrm{C} 7-\mathrm{C} 6-\mathrm{C} 8-\mathrm{C} 9$ & $33.3(2)$ & $\mathrm{C} 6-\mathrm{C} 5-\mathrm{C} 27-\mathrm{C} 32$ & $-163.87(12)$ \\
\hline $\mathrm{C} 5-\mathrm{C} 6-\mathrm{C} 8-\mathrm{C} 9$ & $-155.55(13)$ & $\mathrm{C} 4-\mathrm{C} 5-\mathrm{C} 27-\mathrm{C} 32$ & $67.74(16)$ \\
\hline $\mathrm{O} 1-\mathrm{C} 8-\mathrm{C} 9-\mathrm{C} 14$ & $-144.49(14)$ & $\mathrm{C} 32-\mathrm{C} 27-\mathrm{C} 28-\mathrm{C} 29$ & $-0.3(2)$ \\
\hline $\mathrm{C} 6-\mathrm{C} 8-\mathrm{C} 9-\mathrm{C} 14$ & $31.6(2)$ & $\mathrm{C} 5-\mathrm{C} 27-\mathrm{C} 28-\mathrm{C} 29$ & $-179.83(14)$ \\
\hline $\mathrm{O} 1-\mathrm{C} 8-\mathrm{C} 9-\mathrm{C} 10$ & $31.66(19)$ & $\mathrm{C} 27-\mathrm{C} 28-\mathrm{C} 29-\mathrm{C} 30$ & $0.6(2)$ \\
\hline $\mathrm{C} 6-\mathrm{C} 8-\mathrm{C} 9-\mathrm{C} 10$ & $-152.29(14)$ & $\mathrm{C} 28-\mathrm{C} 29-\mathrm{C} 30-\mathrm{C} 31$ & $-0.7(2)$ \\
\hline $\mathrm{C} 14-\mathrm{C} 9-\mathrm{C} 10-\mathrm{C} 11$ & $-2.0(2)$ & $\mathrm{C} 29-\mathrm{C} 30-\mathrm{C} 31-\mathrm{C} 32$ & $0.6(2)$ \\
\hline $\mathrm{C} 8-\mathrm{C} 9-\mathrm{C} 10-\mathrm{C} 11$ & $-178.33(14)$ & $\mathrm{C} 30-\mathrm{C} 31-\mathrm{C} 32-\mathrm{C} 27$ & $-0.3(2)$ \\
\hline $\mathrm{C} 9-\mathrm{C} 10-\mathrm{C} 11-\mathrm{C} 12$ & $1.4(3)$ & $\mathrm{C} 28-\mathrm{C} 27-\mathrm{C} 32-\mathrm{C} 31$ & $0.2(2)$ \\
\hline $\mathrm{C} 10-\mathrm{C} 11-\mathrm{C} 12-\mathrm{C} 13$ & $0.2(3)$ & $\mathrm{C} 5-\mathrm{C} 27-\mathrm{C} 32-\mathrm{C} 31$ & $179.72(13)$ \\
\hline $\mathrm{C} 11-\mathrm{C} 12-\mathrm{C} 13-\mathrm{C} 14$ & $-1.2(2)$ & $\mathrm{C} 6-\mathrm{C} 7-\mathrm{N} 1-\mathrm{C} 1$ & $-148.49(13)$ \\
\hline
\end{tabular}




$\begin{array}{llll}\mathrm{C} 12-\mathrm{C} 13-\mathrm{C} 14-\mathrm{C} 9 & 0.6(2) & \mathrm{C} 15-\mathrm{C} 7-\mathrm{N} 1-\mathrm{C} 1 & 31.67(18) \\ \mathrm{C} 10-\mathrm{C} 9-\mathrm{C} 14-\mathrm{C} 13 & 1.0(2) & \mathrm{C} 6-\mathrm{C} 7-\mathrm{N} 1-\mathrm{C} 3 & 0.4(2) \\ \mathrm{C} 8-\mathrm{C} 9-\mathrm{C} 14-\mathrm{C} 13 & 177.12(13) & \mathrm{C} 15-\mathrm{C} 7-\mathrm{N} 1-\mathrm{C} 3 & -179.42(11) \\ \mathrm{N} 1-\mathrm{C} 7-\mathrm{C} 15-\mathrm{C} 20 & 50.14(18) & \mathrm{C} 2-\mathrm{C} 1-\mathrm{N} 1-\mathrm{C} 7 & 130.66(13) \\ \mathrm{C} 6-\mathrm{C} 7-\mathrm{C} 15-\mathrm{C} 20 & -129.69(15) & \mathrm{C} 2-\mathrm{C} 1-\mathrm{N} 1-\mathrm{C} 3 & -21.25(14) \\ \mathrm{N} 1-\mathrm{C} 7-\mathrm{C} 15-\mathrm{C} 16 & -130.46(14) & \mathrm{N} 2-\mathrm{C} 3-\mathrm{N} 1-\mathrm{C} 7 & -152.67(12) \\ \mathrm{C} 6-\mathrm{C} 7-\mathrm{C} 15-\mathrm{C} 16 & 49.7(2) & \mathrm{C} 21-\mathrm{C} 3-\mathrm{N} 1-\mathrm{C} 7 & 89.46(14) \\ \mathrm{C} 20-\mathrm{C} 15-\mathrm{C} 16-\mathrm{C} 17 & 1.5(2) & \mathrm{C} 4-\mathrm{C} 3-\mathrm{N} 1-\mathrm{C} 7 & -36.33(16) \\ \mathrm{C} 7-\mathrm{C} 15-\mathrm{C} 16-\mathrm{C} 17 & -177.90(14) & \mathrm{N} 2-\mathrm{C} 3-\mathrm{N} 1-\mathrm{C} 1 & 0.10(14) \\ \mathrm{C} 15-\mathrm{C} 16-\mathrm{C} 17-\mathrm{C} 18 & 0.0(3) & \mathrm{C} 21-\mathrm{C} 3-\mathrm{N} 1-\mathrm{C} 1 & -117.77(12) \\ \mathrm{C} 16-\mathrm{C} 17-\mathrm{C} 18-\mathrm{C} 19 & -1.1(3) & \mathrm{C} 4-\mathrm{C} 3-\mathrm{N} 1-\mathrm{C} 1 & 116.44(12) \\ \mathrm{C} 17-\mathrm{C} 18-\mathrm{C} 19-\mathrm{C} 20 & 0.7(3) & \mathrm{C} 1-\mathrm{C} 2-\mathrm{N} 2-\mathrm{C} 3 & -35.78(14) \\ \mathrm{C} 18-\mathrm{C} 19-\mathrm{C} 20-\mathrm{C} 15 & 0.9(3) & \mathrm{N} 1-\mathrm{C} 3-\mathrm{N} 2-\mathrm{C} 2 & 22.05(14) \\ \mathrm{C} 16-\mathrm{C} 15-\mathrm{C} 20-\mathrm{C} 19 & -1.9(2) & \mathrm{C} 21-\mathrm{C} 3-\mathrm{N} 2-\mathrm{C} 2 & 142.42(11) \\ \mathrm{C} 7-\mathrm{C} 15-\mathrm{C} 20-\mathrm{C} 19 & 177.47(14) & \mathrm{C} 4-\mathrm{C} 3-\mathrm{N} 2-\mathrm{C} 2 & -92.91(13) \\ \end{array}$

Hydrogen-bond geometry $\left(\hat{A},{ }^{\circ}\right)$

$C g 3$ is the centroid of the $\mathrm{C} 9-\mathrm{C} 14$ phenyl ring.

\begin{tabular}{lllll}
\hline$D-\mathrm{H}^{\prime} \cdots A$ & $D-\mathrm{H}$ & $\mathrm{H} \cdots A$ & $D \cdots A$ & $D-\mathrm{H} \cdots A$ \\
\hline $\mathrm{C} 2-\mathrm{H} 2 B \cdots \mathrm{O} 1^{\mathrm{i}}$ & 0.99 & 2.43 & $3.4084(19)$ & 168 \\
$\mathrm{C} 24-\mathrm{H} 24 \cdots C g 3^{\mathrm{ii}}$ & 0.95 & 2.83 & $3.5886(18)$ & 138 \\
\hline
\end{tabular}

Symmetry codes: (i) $-x,-y,-z+2$; (ii) $-x,-y,-z+1$. 